\section{A FACTOR IN HEART MUSCLE REQUIRED FOR THE REDUCTION OF CYTOCHROME C BY CYTOCHROME $b$}

\author{
By E. C. SLATER \\ Molteno Institute, University of Cambridge
}

$\mathbf{T}$ is well established ${ }^{1}$ that the intracellular respir1 atory complex catalysing the aerobic oxidation of succinate (the succinic oxidase system) includes the cytochrome system in addition to the specific enzyme responsible for the activation of succinate (that is, succinic dehydrogenase). Thus the transport of electrons from succinate to molecular oxygen proceeds through a chain of electron carriers of successively higher oxidation-reduction potential2. Four such carriers have been identified spectroscopically, namely, cytochromes $b, c, a$ and $a_{3}$. Cytochrome $a_{3}$ is probably identical with cytochrome oxidase ${ }^{3}$. The purpose of the present communication is to present evidence for the existence of an additional carrier. This evidence was obtained by studying the mechanism of the action of certain reducing agents (particularly the dithiol 2,3 dimercaptopropanol (B.A.L.)) on the succinic oxidase system of heart muscle.

It was found that the oxidation of this dithiol by air at $p H \quad 7 \cdot 1$ was catalysed by the heart-muscle preparation of Keilin and Hartree ${ }^{1}$. Treatment of the heart-muscle preparation with B.A.L., in the presence of air, had two quite distinct effects on the enzymes responsible for the oxidation of succinate : (a) Complete inactivation of the succinic oxidase system (measured by the rate of oxidation of succinate in the presence of excess cytochrome $c$ ), without any effect on the succinic dehydrogenase (measured by the rate of oxidation of succinate in the presence of methylene blue and potassium cyanide); this inactivation is complete soon after the addition of the B.A.L. (b) A partial inhibition of succinic dehydrogenase, which does not occur until more than half the B.A.L. is oxidized; this inhibition is probably largely dure to the action of oxidized B.A.L. on the sulphydryl groups of succinic dehydrogenase (cf. Hopkins and Morgan ${ }^{4}$ ). Incidentally, these observations reconcile the apparently divergent findings of Barron et al. ${ }^{5}$ and of Webb and van Heyningen ${ }^{6}$ on the action of B.A.L. on the succinic oxidase system, since the former workers measured the effect on the complete succinic oxidase system, whereas the latter studied the effect on the succinic dehydrogenase alone.

The inactivation of the complete system without concomitant inhibition of succinic dehydrogenase was produced only if B.A.L. was oxidized in air in the presence of the enzyme. No inhibition resulted from contact with the dithiol under anaerobic conditions, while oxidized B.A.L. only slightly inhibited the succinic oxidase system and at the same time inhibited succinic dehydrogenase. Although it was found that hydrogen peroxide was produced during the oxidation of the B.A.L., the effect on the enzyme was not due non-specifically to this hydrogen peroxide, since hydrogen peroxide produced by other systems (notatin-glucose; $d$-amino-acid oxidase and its substrates) inactivated the enzyme only partially, and this inactivation, but not the B.A.L.-induced inactivation, could be partly protected by alcohol and by pyruvate and was increased by catalase. Moreover, the addition of copper, which increased the rate of oxidation of $B . A . L$. and, therefore, the rate of production of hydrogen peroxide, decreased the degree of inactivation.

Treatment of the heart-muscle preparation with B.A.L. under conditions which led to complete inactivation of the succinic oxidase system had very little effect (about 10 per cent inhibition) on the cytochrome oxidase activity (measured by the rate of oxidation of ascorbic acid, hydroquinone or $p$-phenylene diamine in the presence of excess cytochrome $c$ ). The ability of the heart-muscle preparation to catalyse the oxidation of $p$-phenylene diamine in the absence of added cytochrome $c^{7}$ was inhibited by about 35 per cent by the same treatment.

This treatment with B.A.L. did not affect the intensities of the cytochrome $a+a_{3}, b$ and $c$ bands, after reduction with sodium hydrosulphite, showing that the amounts of these cytochromes were not altered. When, however, succinate was added instead of sodium hydrosulphite, an impairment of the rate of oxidation of cytochrome $b$ by cytochrome $c$ was observed : thus on adding succinate to heart-muscle preparation treated with B.A.L. and shaking with air, the $b$-band alone was visible, that is, in the reduced state (cf. the action of narcotics ${ }^{1}$ ). The cytochrome $b$ could, however, be oxidized by the addition of methylene blue or dichlorphenol indophenol. Although cytochromes $a+a_{3}, b, c$, were not destroyed by B.A.L., the total hæmatin content (measured by the intensity of the pyridine-hæmochromogen band at 548-560 mu after the addition of sodium hydrosulphite and pyridine) was reduced by about 20 per cent when the succinic oxidase system was completely inactivated. Thus treatment with $B . A . L$. destroyed some hæmatin compound the spectrum of which is not visible in the heart muscle preparation, either before or after treatment with hydrosulphite. There was a close correlation between the amount of hæmatin compound destroyed and the

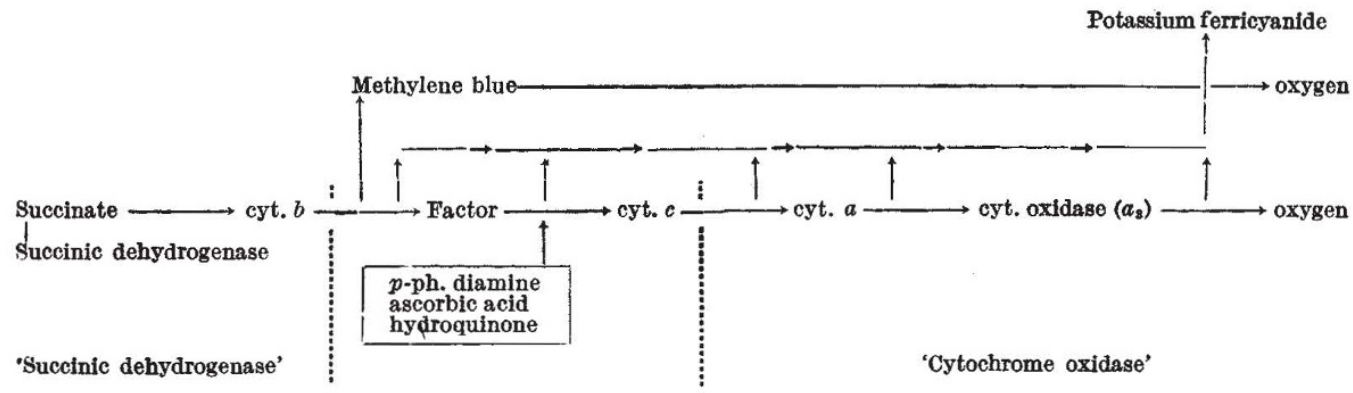

THE COMPONENTS OF THE SUOCTNIO OXIDASE SYSTEM 


\section{A FACTOR IN HEART MUSCLE REQUIRED FOR THE REDUCTION OF CYTOCHROME C BY CYTOCHROME $b$}

\author{
By E. C. SLATER \\ Molteno Institute, University of Cambridge
}

$\mathbf{T}$ is well established ${ }^{1}$ that the intracellular respir1 atory complex catalysing the aerobic oxidation of succinate (the succinic oxidase system) includes the cytochrome system in addition to the specific enzyme responsible for the activation of succinate (that is, succinic dehydrogenase). Thus the transport of electrons from succinate to molecular oxygen proceeds through a chain of electron carriers of successively higher oxidation-reduction potential2. Four such carriers have been identified spectroscopically, namely, cytochromes $b, c, a$ and $a_{3}$. Cytochrome $a_{3}$ is probably identical with cytochrome oxidase ${ }^{3}$. The purpose of the present communication is to present evidence for the existence of an additional carrier. This evidence was obtained by studying the mechanism of the action of certain reducing agents (particularly the dithiol 2,3 dimercaptopropanol (B.A.L.)) on the succinic oxidase system of heart muscle.

It was found that the oxidation of this dithiol by air at $p H \quad 7 \cdot 1$ was catalysed by the heart-muscle preparation of Keilin and Hartree ${ }^{1}$. Treatment of the heart-muscle preparation with B.A.L., in the presence of air, had two quite distinct effects on the enzymes responsible for the oxidation of succinate : (a) Complete inactivation of the succinic oxidase system (measured by the rate of oxidation of succinate in the presence of excess cytochrome $c$ ), without any effect on the succinic dehydrogenase (measured by the rate of oxidation of succinate in the presence of methylene blue and potassium cyanide); this inactivation is complete soon after the addition of the B.A.L. (b) A partial inhibition of succinic dehydrogenase, which does not occur until more than half the B.A.L. is oxidized; this inhibition is probably largely dure to the action of oxidized B.A.L. on the sulphydryl groups of succinic dehydrogenase (cf. Hopkins and Morgan ${ }^{4}$ ). Incidentally, these observations reconcile the apparently divergent findings of Barron et al. ${ }^{5}$ and of Webb and van Heyningen ${ }^{6}$ on the action of B.A.L. on the succinic oxidase system, since the former workers measured the effect on the complete succinic oxidase system, whereas the latter studied the effect on the succinic dehydrogenase alone.

The inactivation of the complete system without concomitant inhibition of succinic dehydrogenase was produced only if B.A.L. was oxidized in air in the presence of the enzyme. No inhibition resulted from contact with the dithiol under anaerobic conditions, while oxidized B.A.L. only slightly inhibited the succinic oxidase system and at the same time inhibited succinic dehydrogenase. Although it was found that hydrogen peroxide was produced during the oxidation of the B.A.L., the effect on the enzyme was not due non-specifically to this hydrogen peroxide, since hydrogen peroxide produced by other systems (notatin-glucose; $d$-amino-acid oxidase and its substrates) inactivated the enzyme only partially, and this inactivation, but not the B.A.L.-induced inactivation, could be partly protected by alcohol and by pyruvate and was increased by catalase. Moreover, the addition of copper, which increased the rate of oxidation of $B . A . L$. and, therefore, the rate of production of hydrogen peroxide, decreased the degree of inactivation.

Treatment of the heart-muscle preparation with B.A.L. under conditions which led to complete inactivation of the succinic oxidase system had very little effect (about 10 per cent inhibition) on the cytochrome oxidase activity (measured by the rate of oxidation of ascorbic acid, hydroquinone or $p$-phenylene diamine in the presence of excess cytochrome $c$ ). The ability of the heart-muscle preparation to catalyse the oxidation of $p$-phenylene diamine in the absence of added cytochrome $c^{7}$ was inhibited by about 35 per cent by the same treatment.

This treatment with B.A.L. did not affect the intensities of the cytochrome $a+a_{3}, b$ and $c$ bands, after reduction with sodium hydrosulphite, showing that the amounts of these cytochromes were not altered. When, however, succinate was added instead of sodium hydrosulphite, an impairment of the rate of oxidation of cytochrome $b$ by cytochrome $c$ was observed : thus on adding succinate to heart-muscle preparation treated with B.A.L. and shaking with air, the $b$-band alone was visible, that is, in the reduced state (cf. the action of narcotics ${ }^{1}$ ). The cytochrome $b$ could, however, be oxidized by the addition of methylene blue or dichlorphenol indophenol. Although cytochromes $a+a_{3}, b, c$, were not destroyed by B.A.L., the total hæmatin content (measured by the intensity of the pyridine-hæmochromogen band at 548-560 mu after the addition of sodium hydrosulphite and pyridine) was reduced by about 20 per cent when the succinic oxidase system was completely inactivated. Thus treatment with $B . A . L$. destroyed some hæmatin compound the spectrum of which is not visible in the heart muscle preparation, either before or after treatment with hydrosulphite. There was a close correlation between the amount of hæmatin compound destroyed and the

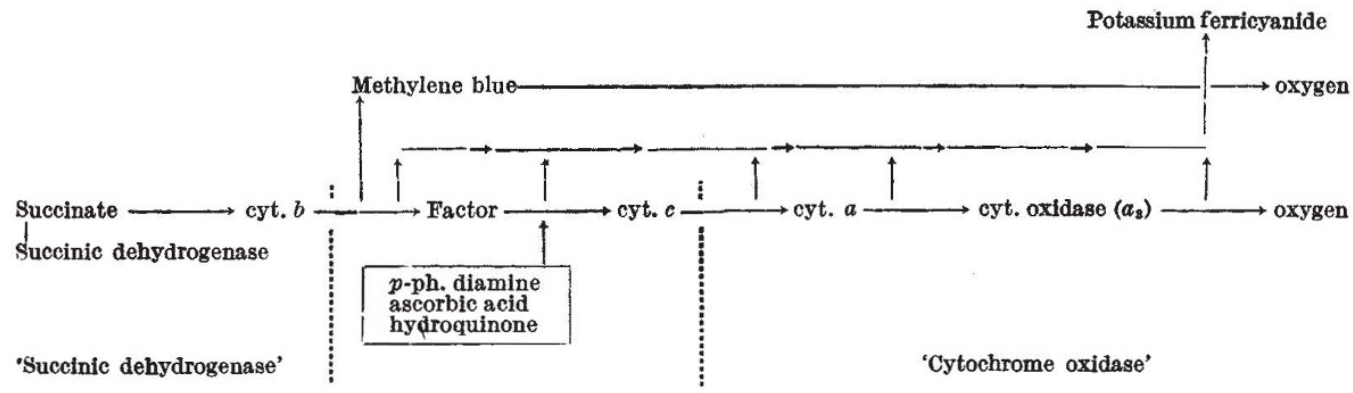

THE COMPONENTS OF THE SUOCTNIO OXIDASE SYSTEM 
degree of inactivation of the succinic oxidase system when different concentrations of B.A.L. were allowed to act on the enzyme for various times.

The enzyme preparation after treatment with B.A.L. could not be reactivated by catalase, cytochrome $c$, serum proteins, denatured globin, calcium phosphate gel, Straub's ' $S C$ factor'8, copper, aqueous extract of fresh heart-muscle mince (rich in myoglobin) or various fractions of the heart muscle preparation. The enzyme could, however, be protected from the action of B.A.L. by the presence, during treatment, of cytochrome $c$, which caused rapid oxidation (through the cytochrome oxidase system) of the dithiol.

Treatment with B.A.L. under conditions which caused complete inactivation of the succinic oxidase system, with about 10 per cent inhibition of succinic dehydrogenase, inhibited the activity of the system, measured anaerobically by the rate of reduction of ferricyanide, by 35 per cent.

These observations suggest the existence of a B.A.L.-labile factor, probably a hæmatin compound, which transfers electrons from cytochrome $b$ to cytochrome $c$ and is destroyed by coupled oxidation with reducing agents (cf. the action of ascorbic acid on hæmoglobin, Lemberg et al. ${ }^{9}$ ) when the heart-muscle preparation is treated with reducing agents in the presence of air.

The relationships between this factor and the cytochromes are shown in the accompanying scheme, the arrows showing direction of electron transfer. Thus destruction of the factor would not affect the oxidation of succinate through methylene blue as carrier, nor the oxidation of various reducing agents through eytochromes $c$ and $a$ and cytochrome oxidase; but would prevent completely the transfer of electrons from cytochrome $b$ to cytochrome $c$ and thence to molecular oxygen. It is postulated that potassium ferricyanide, with an oxidation-reduction potential $\left(E^{\prime}{ }_{0}\right.$ at $p \mathrm{H}$ $7 \cdot 3=+0 \cdot 41$ V.) much higher than that of succinate $\rightleftharpoons$ fumarate $\left(E^{\prime}{ }_{0}\right.$ at $p \mathrm{H}, 7 \cdot 3=-0.01 \mathrm{~V}$.) or of cytochrome $b\left(E^{\prime}{ }_{0}\right.$ at $p H 7 \cdot 3=-0.04 \mathrm{~V}$.) operates partly directly with cytochrome $b$ and partly through the B.A.L.-labile factor or the cytochromes of higher oxidation-reduction potential $\left(E^{\prime}{ }_{0}\right.$ of cytochrome $c=+0.26 \mathrm{~V}$. , cytochrome $a=+0.29$ V.). Thus the destruction of the factor causes a partial, but not complete, inhibition of the rate of oxidation by ferricyanide.

It is possible that the factor is the same as, or closely related to, the component $X$ suggested by Bach et al. ${ }^{10}$ to be necessary for the reduction of cytochrome $c$ by cytochrome $b_{2}$ in yeast.

The complete experimental data and a discussion of these findings in relation to previous work on the succinic oxidase system will appear elsewhere.

I would like to express my sincere thanks to Prof. D. Keilin for his most valuable advice throughout this investigation. I also wish to thank the British Council for a scholarship.

${ }^{2}$ Keilin, D., and Hartree, E. F., Proc. Roy. Soc., B, 129, 277 (1940). Ball, E. G., Biochem. Z., 295, 262 (1938).

Keilin, D., and Hartree, E. F., Proc. Roy. Soc., B, 127, 167 (1939).

'Hopkins, F. G., and Morgan, E. J., Biochem. J., 32, 611 (1938).

- Barron, E. S. G., Miller, Z. B., and Meyer, J., Biochem. J., 41, 78 (1947).

- Webb, E. C., and van Heyningen, R., Biochem. J., 41, 74 (1947).

'Keilin, D., and Hartree, E. F., Proc. Roy. Soc., B, 125, 171 (1938).

- Straub, F. B., Z. physiol. Chem., 272, 219 (1942).

- Lemberg, R.. Legge, J. W. and Lockwood, W. H., Biochem. J., 35, 328, 339. 353, 363 (1941).

${ }^{10}$ Bach, S. J., Dixon, M., and Zerfas, L. G., Biochem. J., 40, 229 (1946).

\section{PLANT ANALYSIS AND FERTILIZER REQUIREMENTS}

A QUICK and reliable method of determining the fertilizer requirements of crops and of discovering what nutrient deficiency is responsible for the unsatis. factory growth of some particular crop would be of immense value in advisory work. The problem can be approached from several different points of view, such as soil analysis, field trials, pot cultures, leaf injection, plant analysis and the use of visual symptoms. The relative advantages and disadvantages of these various methods of diagnosing fertilizer requirements are briefly but clearly summarized in the paper under notice*, and an extensive list of references facilitates fuller study of the individual methods.

A short historical account of the early work on diagnostic plant analysis reminds the reader that there is much to be learnt from these early papers. Developments during the past twenty-five years are discussed in more detail, and a great deal of information on the content of nutrients in plant material in relation to the incidence of deficiency or toxicity symptoms is summarized in tabular form.

Reference is also made to the use of plant analysis in the determination of current nutrient requirements by so-called 'tissue tests'. Carried out on sap or extracts of fresh material, it is suggested that the fractions estimated by these tissue tests are mainly unassimilated material which has recently entered the plant and that their concentration thus represents the current rate of nutrient intake. The rapidity with which 'tissue tests' can be performed and the fact that their results can be translated into immediate fertilizer treatment quickly enough to be effective even on annual crops, provided the problem is recognized early in the life of the crop, makes them specially attractive in advisory work. If they can be shown to be reasonably reliable and their interpretation does not require the establishment of a very elaborate series of standards, they seem likely to displace some of the older methods used in advisory work.

As the authors of this paper point out, when defining the aim of a method of deficiency diagnosis, there is an important distinction between methods to be used to improve the nutrition, during the course of its growth, of the crop analysed, and those where the plants analysed serve only as an index of the state of the soil and as a guide to the best fertilizer treatment for subsequent crops.

In a discussion of the relation of internal nutrient concentration to nutrient supply, evidence from barley grown in sand cultures, to which nutrients were added before germination, is used to show that: (1) the concentration in the tissues will depend upon the time during development at which determinations are made; and (2) the results will depend upon the part of the plant chosen for analysis. Analysis of the plant soon after germination (when the seed reserves are exhausted) will give some measure of the concentration of the nutrients in the rooting medium, especially in plants like cereals that branch very rapidly in the early stages.

The bearing of these facts on the relations to be expected with plants growing in the field are complic. ated by the difference in the mode of supply of the nutrient; for not only are the differences in the

* Chemical Composition of Plants as an Index of their Nutritional Status. (Tech. Comm. No. 17, Imperial Bureau of Horticulture and Plantation Crops.) By Dr. D.' W. Goodall and Dr. F. G. Gregory. Pp. 167. (Aberystwyth : Imp. Bur., 1947.) 98 . 